\title{
Cryoablation with drug-loaded bead embolization in the treatment of unresectable hepatocellular carcinoma: safety and efficacy analysis
}

\author{
Jian-Ying Zeng ${ }^{1}$, Xiang-Hao Piao ${ }^{2}$, Zhong-Yuan Zou ${ }^{2}$, Qing-Feng Yang ${ }^{2}$, Zi-Lin Qin ${ }^{3}$, \\ Ji-Bing Chen ${ }^{1}$, Liang Zhou ${ }^{4}$, Li-Zhi Niu${ }^{4}$ and Jian-Guo Liu ${ }^{4}$ \\ ${ }^{1}$ Central Laboratory, Affiliated Fuda Cancer Hospital, Jinan University, Guangzhou 510665, P.R. China \\ ${ }^{2}$ Department of Intervention, Affiliated Fuda Cancer Hospital, Jinan University, Guangzhou 510665, P.R. China \\ ${ }^{3}$ School of Medicine, Jinan University, Guangzhou 510632, P.R. China \\ ${ }^{4}$ Department of Surgical Oncology, Affiliated Fuda Cancer Hospital, Jinan University, Guangzhou 510665, P.R. China \\ Correspondence to: Xiang-Hao Piao, email: kzgdo-01@163.com \\ Keywords: hepatocellular carcinoma; transarterial chemoembolization; cryoablation; hepasphere microsphere; doxorubicin \\ Received: November 02, $2017 \quad$ Accepted: January 03, $2018 \quad$ Published: January 08, 2018 \\ Copyright: Zeng et al. This is an open-access article distributed under the terms of the Creative Commons Attribution License 3.0 \\ (CC BY 3.0), which permits unrestricted use, distribution, and reproduction in any medium, provided the original author and source \\ are credited.
}

\section{ABSTRACT}

This study aimed to explore the efficacy and safety of drug-eluting bead (DEB) embolization (DEB-TACE) when combined with cryoablation in the treatment of unresectable hepatocellular carcinoma (HCC). The study was a single-center randomized controlled trial comprised of 60 patients with HCC conducted between August 2015 and October 2017. The patients were randomly divided into two groups: DEB-TACE combined with cryoablation (DEB-TACE-Cryo group) or cryoablation alone (Cryo group). Inter-group differences in overall survival, progression-free survival, and adverse reactions were assessed. The operative success rates were $82.7 \%$ and $\mathbf{7 7 . 4 \%}$ in the DEB-TACE-Cryo group and Cryo group, respectively, with no operative mortality. The overall survival and progression-free survival in the DEB-TACE-Cryo group were significantly higher than those in the Cryo group (16.8 months vs.13.4 months, $P=0.0493 ; 8.1$ months vs. 6.0 months, $P=0.0089$, respectively). The postoperative complications in the two groups were rated as grade 1 or grade 2 , according to guidelines set by the National Cancer Institute Common Terminology Criteria for Adverse Events Version 4.0 (CTCAE V4.0). We demonstrated that DEB-TACE combined with cryoablation was effective, well tolerated, and had a low complication rate. Therefore, this combination therapy may be a better choice for the treatment of unresectable hepatocellular carcinoma.

\section{INTRODUCTION}

Worldwide, hepatocellular carcinoma (HCC) is the second leading cause of cancer death in men and the sixth in women. During 2012, an estimated 782,500 new HCC cancer cases were diagnosed; However, due to very poor prognosis, the number of deaths was almost the same $(745,500)[1]$. For very early stage and early stage patients, surgical resection, radiofrequency ablation (RFA), and/or liver transplantation are effective options for treatment [2]. Unfortunately, nearly $30 \%$ of patients who have been diagnosed at intermediate or advanced stages have no surgical options [3-4]. Most patients diagnosed with unresectable HCC succumb to liver failure as a result of advancing cirrhosis or tumor progression [5]. Transarterial chemoembolization (TACE) and sorafenib are recommended palliative therapies for patients with unresectable HCC [2]. However, the efficacy of TACE depends on the size of the tumor. For tumors larger than $5 \mathrm{~cm}$ in diameter, the rate of complete necrosis is low 
and the long-term outcome is negatively affected [6]. Accordingly, TACE combined with other therapies, such as local liver ablation, has been widely applied and proven to significantly improve tumor response and patient survival rates [7-11]. Cryoablation is another alternative local treatment option for unresectable HCC. Compared to RFA, cryoablation has several unique advantages including more clearly visualized margins of ablated lesions from normal tissue on ultrasound (USG) or computed tomography (CT), larger range of ablation zones, less pain and tumor immunosuppressive effect [12-16]. It has been demonstrated that cryoablation combined with conventional TACE treatment can further improve on the therapeutic effect of cryoablation alone against $\mathrm{HCC}$ [17-21]. However, the safety and efficacy of TACE with drug-eluting beads (DEBs) combined with cryoablation for HCC have not yet been reported. DEBs are a relatively novel drug delivery embolization system that facilitate anticancer agent release and fixed dosing [22-25]. HepaSphere microspheres, commercially available DEBs, are biocompatible, hydrophilic, non-resorbable, expandable, and conformable microspheres designed for controlled, targeted embolization. They have been shown to be highly effective in the treatment of HCC [26-29].

With the above in mind, this randomized controlled clinical trial combined cryoablation with DEB-TACE, using 50-100 $\mu \mathrm{m}$ diameter HepaSphere microspheres for the treatment of unresectable HCC. The aim of this study was to assess the overall survival, progression-free survival, and adverse events after combined therapy, with a view of achieving a safer and more effective treatment method for unresectable HCC.

\section{RESULTS}

\section{Clinical data}

From August 2015 to September 2016, 397 patients received liver tumor treatment in our hospital. 290 cases were diagnosed as HCC. In accordance with the eligibility criteria and exclusion criteria, 73 patients with portal vein embolization, 59 patients with extrahepatic multiple metastases, 34 patients with obvious hepatic artery-portal vein fistulas or artery-venous fistulas, 8 patients with coagulation dysfunction, and 24 patients with severe liver dysfunction were excluded. Additionally, 32 people refused to participate in the study. Ultimately, there were 60 patients with HCC who met the inclusion criteria and agreed to study enrollment. The clinical characteristics for the 60 patients are listed in Table 1 .

\section{Treatment success rate and tumor response}

Four to six weeks after treatment, 5 patients and 7 patients with residual active tumors were identified in the DEB-TACE-Cryo group and Cryo group, and the intervention success rates were $82.7 \%(24 / 29)$ and $77.4 \%$ (24/31), respectively. All 8 patients with residual active tumors underwent a second cryoablation cycle (Figure 1). Six months and 1 year after treatment, there were 5 and 11 cases with local recurrence or distant metastasis in the DEB-TACE-Cryo group, respectively. This recurrence rate represents $17.2 \%$ and $37.9 \%$ (7 local recurrence and 9 distant metastasis), respectively. In the Cryo group, there were 7 cases and 21 cases with local recurrences or metastases, and the recurrence rates were $22.6 \%$ and $67.7 \%$ (8 local recurrences, 13 distant metastases), respectively. The difference in the recurrence rate of the two groups one year after treatment was statistically significant $(P=0.0207)$.

\section{Survival analysis}

At the time of censor, 14 and 21 patients had expired in the DEB-TACE-Cryo group and Cryo group $(P=$ $0.1264)$, respectively. The median follow-up was $17.2 \pm$ 5.5 months (range, 6 to 26.4 months).

The $0.5,1$, and 2 year overall survival rates in the DEB-TACE-Cryo group and Cryo group were 100\%, $89.7 \%$, and $62.1 \%$, and $100 \%, 83.9 \%$, and $41.9 \%$, respectively (Figure 2A). The median overall survival in the DEB-TACE-Cryo group (16.8 months) was longer than that in the Cryo group (13.4 months), the difference was statistically significant (Hazard Ratio $=0.557 ; 95 \%$ $\mathrm{Cl}, 0.284$ to $1.093 ; P=0.0493$ ).

The 0.5 , and 1 year progression-free survival percentages for the DEB-TACE-Cryo group and the Cryo group were $82.8 \%$ and $62.1 \%$, and $77.4 \%$ and $32.3 \%$, respectively (Figure $2 \mathrm{~B}$ ). The median progression-free survival in the DEB-TACE-Cryo group (8.1 months) was longer than that in the Cryo group (6.0 months), the difference was statistically significant (Hazard Ratio $=$ $0.367 ; 95 \% \mathrm{Cl}, 0.175$ to $0.771 ; P=0.0089$ ).

The causes of death for 8 patients were tumor progression for seven, liver failure for two, and another disease for one of the DEB-TACE-Cryo group. For the Cryo group, the causes of death for 13 patients were tumor progression for nine, liver failure for two, and other diseases for two $(P=0.7292, P=0.9450$, and $P=0.5937$, respectively).

\section{Complications}

There was a $0 \% 30$ day mortality rate. The common complications in the two groups were as follows: fever; pain; coughing; ascites; nausea; pleural effusion; thrombocytopenia; erythropenia; and elevated blood pressure. The most common complication was pain in both groups. The maximum pain scores in the two groups after treatment were significantly higher than those before treatment ( $P=0.01$ and $P<0.000$, Figure 3$)$. There was no significant difference in incidences of complications 
Table 1: Demographics and tumor parameters of study participants

\begin{tabular}{|c|c|c|c|}
\hline Characteristic & DEB-TACE-Cryo group $(n=29)$ & Cryo group $(n=31)$ & $\boldsymbol{P}$ \\
\hline \multicolumn{4}{|l|}{ Sex } \\
\hline $\mathrm{F}$ & $3(10.3)$ & $5(16.1)$ & 0.5101 \\
\hline M & $26(89.6)$ & $26(83.9)$ & \\
\hline \multicolumn{4}{|l|}{ AJCC stage (2010) } \\
\hline IIIA & $14(48.3)$ & $20(64.5)$ & 0.7189 \\
\hline IIIB & $8(27.6)$ & $6(19.4)$ & \\
\hline IIIC & $4(13.8)$ & $3(9.7)$ & \\
\hline IVA & $3(10.3)$ & $3(9.7)$ & \\
\hline \multicolumn{4}{|l|}{ Viral marker } \\
\hline HBsAg positive & $18(62.1)$ & $20(64.5)$ & 0.9202 \\
\hline Anti-HCV positive & $2(6.9)$ & $2(6.5)$ & \\
\hline \multicolumn{4}{|l|}{ Child-Pugh stage } \\
\hline A & $28(83.3)$ & $30(90.3)$ & 0.9617 \\
\hline B & $1(16.7)$ & $1(9.7)$ & \\
\hline Mean age (years) & $65.3 \pm 12.7$ & $56.5 \pm 11.0$ & 0.0513 \\
\hline \multicolumn{4}{|l|}{ ECOG score } \\
\hline 0 & $11(37.9)$ & $13(41.9)$ & 0.2626 \\
\hline 1 & $12(41.4)$ & $16(51.6)$ & \\
\hline 2 & $6(20.7)$ & $2(6.5)$ & \\
\hline Lesion size $(\mathrm{cm})$ & $7.2 \pm 4.5$ & $6.5 \pm 3.8$ & 0.6310 \\
\hline \multicolumn{4}{|l|}{ AFP, IU/mL } \\
\hline$<200$ & 18 & 16 & 0.4860 \\
\hline $200-400$ & 3 & 2 & \\
\hline$>400$ & 8 & 13 & \\
\hline ALT, U/L & $29.3 \pm 14.2$ & $38.9 \pm 13.5$ & 0.0960 \\
\hline AST, U/L & $50.7 \pm 21.9$ & $42.1 \pm 23.0$ & 0.2642 \\
\hline $\mathrm{TBIL}, \mu \mathrm{mol} / \mathrm{L}$ & $14.9 \pm 6.8$ & $19.3 \pm 9.2$ & 0.1691 \\
\hline ALB, g/L & $33.9 \pm 3.4$ & $37.6 \pm 4.3$ & 0.0507 \\
\hline $28-35$ & 12 & 6 & 0.0628 \\
\hline$>35$ & 17 & 25 & \\
\hline \multicolumn{4}{|l|}{ Tumor type } \\
\hline Single massive & $17(58.6)$ & $25(80.6)$ & 0.0628 \\
\hline Multinodular & $12(41.4)$ & $6(19.4)$ & \\
\hline \multicolumn{4}{|l|}{ Ascites } \\
\hline Yes & 8 & 7 & 0.6545 \\
\hline No & 21 & 24 & \\
\hline \multicolumn{4}{|l|}{ Cancer pain } \\
\hline Yes & $5(17.2)$ & $2(6.4)$ & 0.1933 \\
\hline No & $24(82.8)$ & $29(93.6)$ & \\
\hline
\end{tabular}


between the two groups $(P>0.05$, Table 2$)$. According to CTCAE V4.0, the postoperative complications in the two groups were rated as grade 1 or grade 2 .

\section{Postoperative liver function changes}

Seven days after treatment, the serum alanine aminotransferase (ALT) levels of both the DEB-TACE-
Cryo and the Cryo groups and serum total bilirubin (T.BIL) in the DEB-TACE-Cryo group had increased during the short-term $(P=0.008, P=0.002$, and $P=0.016$, respectively). Interestingly, there was no significant increase recorded for any of these levels 30 days after treatment. No obvious changes in aspartate aminotransferase (AST) or albumin (ALB) levels were observed after treatment (Figure 4).
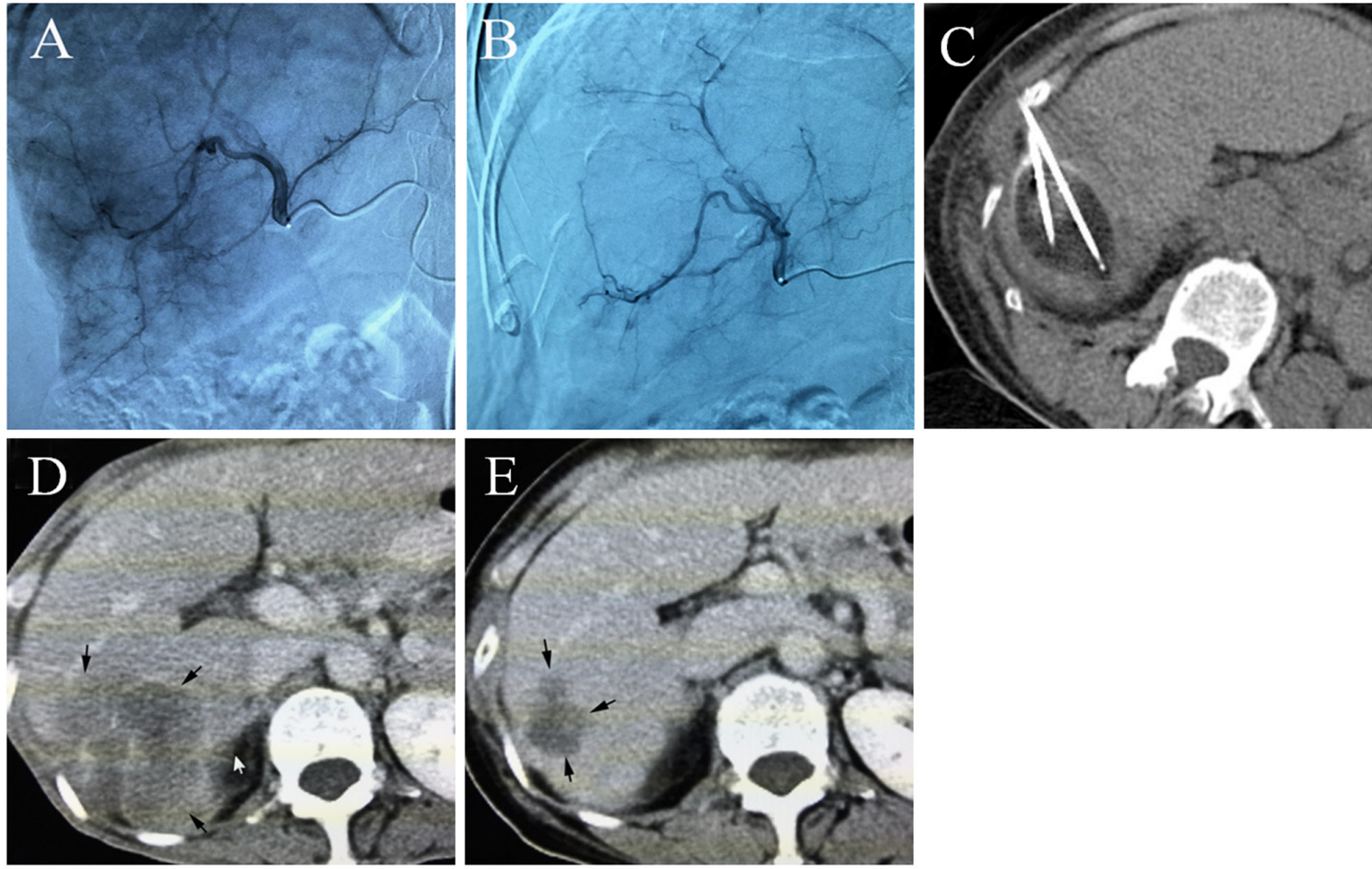

Figure 1: (A) Common artery angiography of a 65 year old male patient with HCC, selective catheterization of the pathologic branch of the right hepatic artery supplying the tumor, (B) the artery angiography shows that most of the tumor staining disappeared after DEB-TACE, (C) percutaneous cryoablation under CT guidance, an ice ball was formed, (D) the contrastenhanced CT scan shows a huge tumor before treatment, (E) five months after DEB-TACE-Cryo treatment, the enhanced CT showed that the tumor was significantly reduced.

A

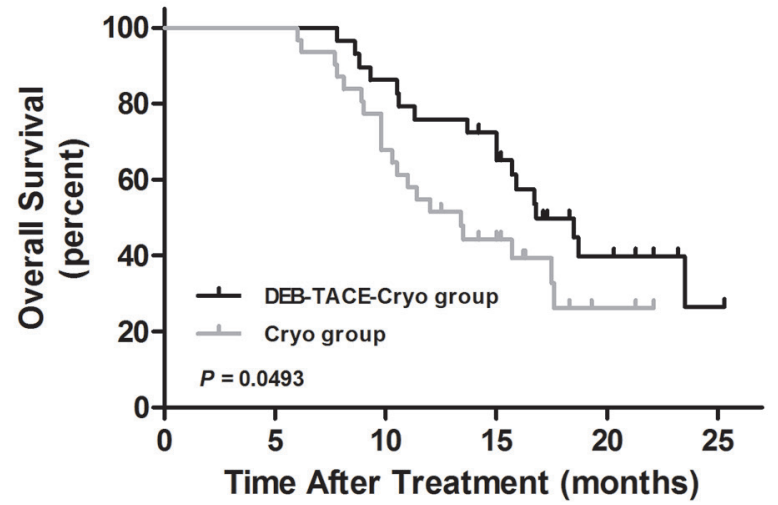

B

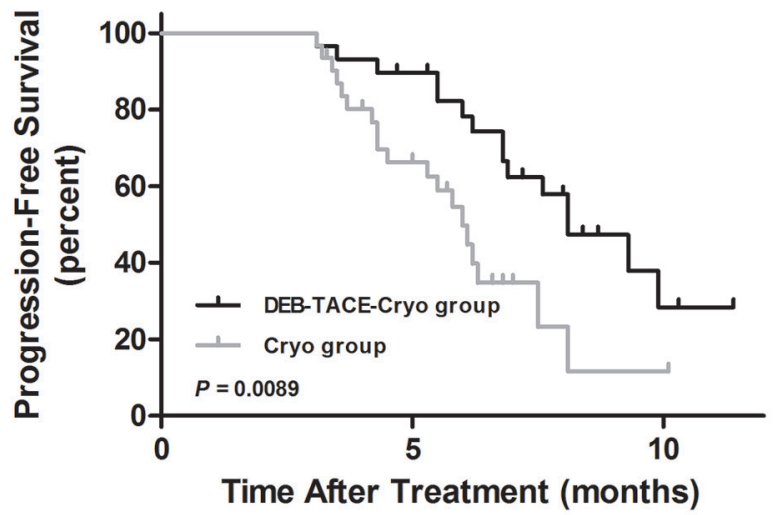

Figure 2: The overall survival curves (A) and progression-free survival curves (B) of transarterial chemoembolization with drug-eluting beads combined with cryoablation (DEB-TACE-Cryo) or cryoablation (Cryo) for unresectable hepatocellular carcinoma (HCC). 
Table 2: Rates of the most common complications after treatment

\begin{tabular}{lccc}
\hline Group & DEB-TACE-Cryo group & Cryo group & $\boldsymbol{P}$ \\
\hline Fever & $6(20.7 \%)$ & $2(6.5 \%)$ & 0.1398 \\
Pain & $21(72.4 \%)$ & $17(54.8 \%)$ & 0.1881 \\
Cough & $5(17.2 \%)$ & $3(9.7 \%)$ & 0.4653 \\
Ascites & $3(10.3 \%)$ & $5(16.1 \%)$ & 0.5101 \\
Nausea & $1(3.4 \%)$ & $1(3.2 \%)$ & 0.5144 \\
Pleural effusion & $5(17.2 \%)$ & $5(16.1 \%)$ & 0.5101 \\
Thrombocytopenia & $3(10.3 \%)$ & $5(16.1 \%)$ & 0.5101 \\
Erythropenia & $3(10.3 \%)$ & $1(3.2 \%)$ & 0.2693 \\
Elevated blood pressure & $3(10.3 \%)$ & $4(12.9 \%)$ & 0.7577 \\
\hline
\end{tabular}

\section{DISCUSSION}

According to follow-up results, both the treatment success rate and short-term tumor response in the DEB-TACE-Cryo group were higher than the Cryo group.The DEB-TACE-Cryo group also achieved a longer progression-free survival and overall survival than the Cryo Group $(P<0.05)$. Therefore, this study showed that combined therapy with DEB-TACE and cryoablation resulted in better treatment efficacy than single therapy with cryoablation for patients with unresectable HCC.
The combined therapy for DEB-TACE-Cryo group during this trial period resulted in a success rate of $82.7 \%$ (24/29) within 4-6 weeks, and 1 and 2 year survival rates of $89.7 \%$ and $62.1 \%$. Veltri et al. reported similar efficacy when RFA combined with TACE was applied for the treatment of unresectable $\mathrm{HCC}$, with survival rates of $89.7 \%$ after 12 months and $67.1 \%$ after 24 months of treatment [30]. Using a similar RFA combinatory approach, Zhang et al. reported that complete ablation was achieved in $76.2 \%$ of cases, with 1,2 , and 3 year survival rates of $89 \%, 61 \%$, and $43 \%$, respectively [31]. Similarly, Zhao et al. reported a 1 year survival rate of

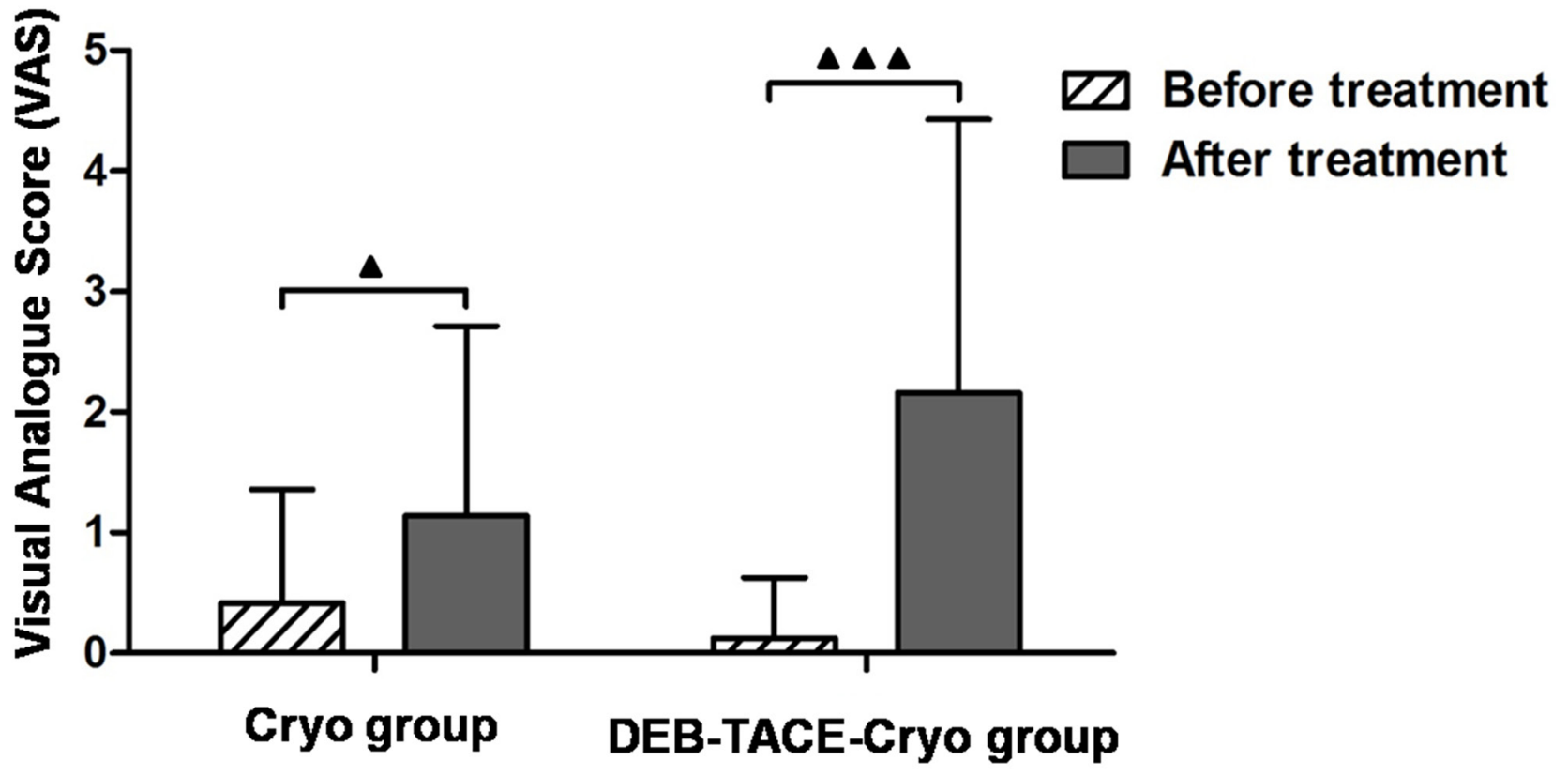

Figure 3: Preoperative and postoperative pain scores in patients with unresectable hepatocellular carcinoma (HCC) treated with transarterial chemoembolization using drug-eluting beads combined with cryoablation (DEB-TACECryo) or cryoablation (Cryo). 
$88.5 \%$ [32]. Considering these comparative results, we believe DEB-TACE-Cryo treatment for HCC may be considered as an effective palliative treatment option for patients with unresectable HCC.

There are several reasons for performing DEBTACE before cryoablation. Firstly, DEB-TACE embolizes tumor internal blood vessels and reduces the heat-drop effect when the ice ball meets the blood vessel, thus promoting the rate of tumor necrosis. Secondly, the local ablation rate is related to the size of the tumor[19, 33]. As DEB-TACE can cause tumor ischemia thus reducing the tumor oxygen supply and release of chemotherapeutic drugs from microspheres in a sustained fashion over a prolonged period of time, it can effectively inactivate the tumor [34-35], which reduces the tumor volume, and increases the rate of complete ablation through cryoablation. Thirdly, DEB-TACE before cryoablation controls intrahepatic micro-lesions, which inhibit recurrences after treatment.

From a safety aspect, the postoperative complications of the two groups were classified as grade 1 or 2 according to the CTCAE V4.0 standard, neither of which is considered to be very severe. There was no significant difference in the incidence of complications between the two groups $(P>0.05)$, which showed that combination therapy did not cause the superposition effects or increases incidence rate of complication. Notably, according to the liver function results in this study, the serum ALT levels in the two groups and the T.BIL in the DEB-TACE-Cryo group increased during the first 7 days. However, there was no significant increase recorded 30 days after treatment. This indicates that neither treatment approach resulted in long-term liver function effects and that both the combined therapy and cryoablation alone are safe for unresectable HCC treatment.

In conclusion, DEB-TACE using HepaSphere microspheres combined with cryoablation may be a more effective approach to improve the outcome for patients with unresectable HCC than cryoablation alone. Use of DEB-TACE using HepaSphere microspheres combined with cryoablation could represent a valuable therapeutic option in unresectable HCC patients. However, since this study is a single-center, small sample randomized clinical trial, we believe multicenter and large-scale clinical trials are warranted to increase the objective accuracy of data in the future.
A

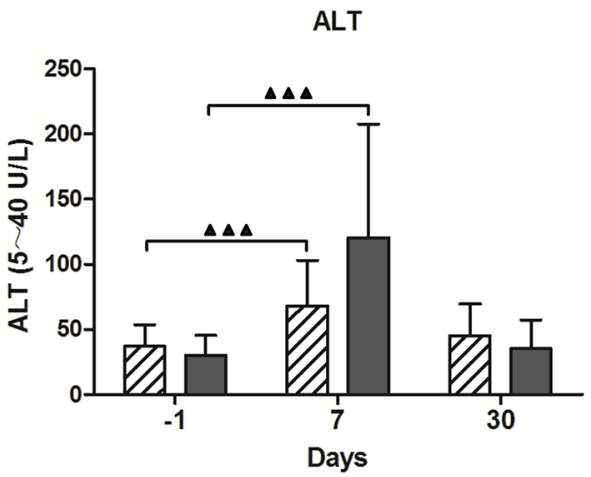

C

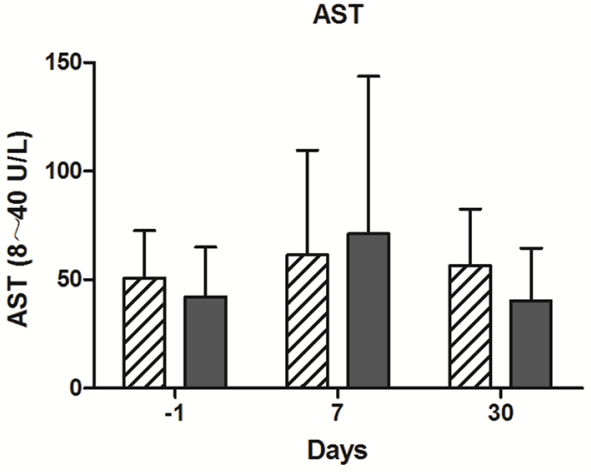

B

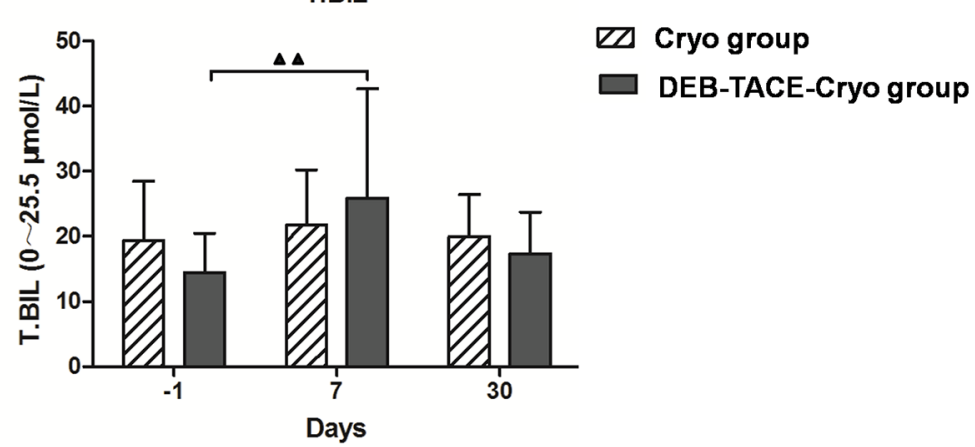

D

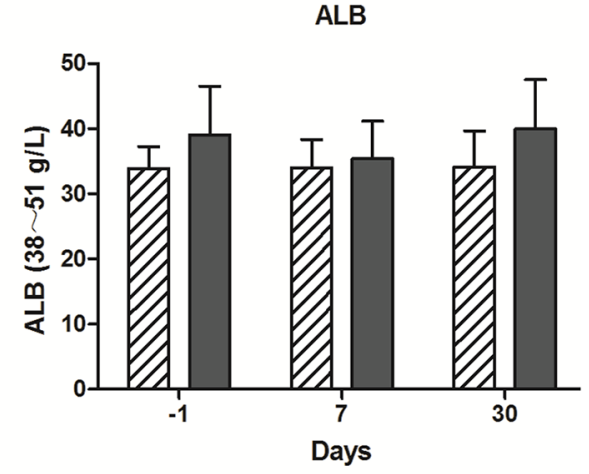

Figure 4: Changes in hepatic functional reserve before and after treatment in both groups. In the DEB-TACE-Cryo group, the serum ALT and TBIL levels were $29.3 \pm 14.2 \mathrm{U} / \mathrm{L}$ and $14.9 \pm 6.77 \mu \mathrm{mol} / \mathrm{L}$, respectively. Seven days after treatment, the ALT and TBIL values increased significantly (to $114.6 \pm 92.1 \mathrm{U} / \mathrm{L}$ and $24.0 \pm 15.4 \mu \mathrm{mol} / \mathrm{L}$, respectively, $P=0.002$ and $P=0.016 ; \mathbf{A}$ and B). In the Cryo group, the ALT levels were $38.9 \pm 13.5 \mathrm{U} / \mathrm{L}$. Seven days after treatment, the ALT values increased significantly (to $73.2 \pm 30.1 \mathrm{U} / \mathrm{L}, P=$ 0.008 ; A). No obvious change in AST or ALB levels were observed after treatment $(P>0.05$; C and D). 


\section{MATERIALS AND METHODS}

\section{Ethics}

The study protocol received ethical approval from the Regional Ethics Committee of the Guangzhou Fuda Cancer Hospital, China. Written informed consent was obtained from each participant, in accordance with the Declaration of Helsinki.

\section{Patient selection}

This prospective randomized controlled study was registered at clinicaltrials.gov [identification number NCT02545556] and conducted in the central laboratory of The Affiliated Fuda Cancer Hospital, Jinan University, Guangzhou, China from August 2015 to October 2017.

According to eligibility and inclusion criteria, 60 patients were enrolled in the study and randomly divided into two groups using a stratified randomization algorithm: the DEB-TACE-Cryo group (29 patients), treated with HepaSphere drug loaded microsphere embolization combined with cryoablation; and the Cryo group (31 patients), subjected to cryoablation alone. Randomization was stratified according to two variables: sex and tumor staging.

For all patients, the diagnosis of $\mathrm{HCC}$ was based on the diagnostic criteria used by the European Association for the Study of the Liver [36]: (1) pathological diagnostic criteria: the biopsy specimens were confirmed by histopathological examination $(\mathrm{n}=43)$; (2) clinical diagnostic criteria: with cirrhosis or hepatitis $\mathrm{B}$ virus (HBV) and/or hepatitis C virus (HCV) infection, the characteristics of typical HCC imaging and/or serum $\alpha$-fetoprotein (AFP) $\geq 400 \mathrm{IU} / \mathrm{mL}$ for 1 month or $\geq 200$ $\mathrm{IU} / \mathrm{mL} \mathrm{ug} / \mathrm{L}$ for 2 months $(\mathrm{n}=17)$.

The eligibility criteria were as follows: (1) Eastern Cooperative Oncology Group (ECOG) score $\leq$ 2 ; (2) platelet count $\geq 100 \times 10^{9} / \mathrm{L}$; (3) white blood cell count $\geq 3 \times 10^{9} / \mathrm{L}$; (4) neutrophil count $\geq 2 \times 10^{9} / \mathrm{L}$; (5) hemoglobin $\geq 90 \mathrm{~g} / \mathrm{L} ;(6)$ prothrombin time international normalized ratio $\geq 1.5$; (7) hepatic tumor not obviously invading the gallbladder, diaphragm or large vessels; (8) absence of level 3 hypertension, severe coronary disease, myelosuppression, respiratory disease, and acute or chronic infection; (9) Child-Pugh score A or B; (10) renal function (serum creatinine $<130 \mu \mathrm{M}$, serum urea $<10$ $\mathrm{mM})$.

The exclusion criteria were (1) Child-Pugh score C; (2) the main portal vein was completely embolized by tumor thrombus, and lack of collateral vessels; (3) multiple distant metastases, estimated survival time of less than three months; (4) proportion of the total liver tumor was more than 70\% cancer; (5) obvious hepatic arteryportal vein fistula, or artery-venous fistula.

\section{TACE with HepaSphere}

\section{Preparation of HepaSphere microspheres}

Preparation was performed as suggested by the manufacturer. Each vial of 50-100 $\mu \mathrm{m}$ HepaSpheres (HepaSphere ${ }^{\mathrm{TM}}$, South Jordan, Utah, USA) was loaded with $25 \mathrm{mg}$ of doxorubicin diluted in $20 \mathrm{~mL}$ of $0.9 \% \mathrm{NaCl}$ solution for injection. The $20 \mathrm{~mL}$ of doxorubicin solution was aspirated into two separate $20 \mathrm{~mL}$ syringes. One of the $20 \mathrm{~mL}$ syringes containing $10 \mathrm{~mL}$ of doxorubicin was added to the HepaSphere vial and agitated frequently for $10 \mathrm{~min}$, after which the remaining $10 \mathrm{~mL}$ were added. The vial was agitated every $10-15 \mathrm{~min}$ for $60 \mathrm{~min}$ to complete the ionic bonding of doxorubicin. After the loading period, all the supernatant was extracted, and an equal quantity of nonionic contrast diluted with saline (50:50) was added to form the final suspension for injection. Overall, the final injectable volume from each vial was $30 \mathrm{~mL}$. Before beginning to inject the suspension, $100 \mu \mathrm{m}$ of glyceryl trinitrate (nitrolingual) was injected through the microcatheter at the target vessel to achieve the maximum vessel dilatation, and the microcatheter was flushed with hyperheparinized saline (2.500 IU/500 $\mathrm{mL}$ of flushing saline). Slow, incremental injection of the suspension followed at a rate of $1-3 \mathrm{~mL} / \mathrm{min}$ until obliteration of the neovascularity. When initial stasis had been achieved, a further pause of 3-5 min was allowed to facilitate the redistribution of the spheres within the lesion and the directed distal push of the spheres by blood inflow pressure. After this pause, more embolic material was injected under the same flow conditions. The total number of vials delivered at each session was recorded.

\section{Catheterization}

The interventional procedure was performed in a dedicated interventional angiography suite and followed the classical steps of hepatic artery catheterization and embolization. After administration of local anesthesia in the right groin, a $5 \mathrm{~F}$ sheath was introduced into the right common femoral artery. Selective catheterization of the celiac trunk and superior mesenteric artery was followed by superselective catheterization of the right and left hepatic arteries. Depending on the randomization, HepaSphere microspheres loaded with doxorubicin were administered by a transcatheter technique under fluoroscopic guidance. After administration, a second hepatic angiography was performed to look for a residual vascular tumor blush. 


\section{Cryoablation}

Each procedure comprised two freeze-thaw cycles accomplished using an argon gas-based cryosurgical unit (Endocare, Irvine, CA, USA). Cryoprobes ( $3 \mathrm{~mm}$ in diameter) were inserted into the center of the tumor mass under ultrasonographic guidance, each reaching a temperature of $-150{ }^{\circ} \mathrm{C}$ at the tip of the probe. The duration of freezing depended on the achievement of an ice ball that extended $1 \mathrm{~cm}$ beyond the boundary of the tumor and was visible as a hypoechoic region on ultrasonography. Generally, the maximum freezing time was $15 \mathrm{~min}$, followed by thawing for $5 \mathrm{~min}$; this cycle was then repeated. For masses $<6 \mathrm{~cm}$ in long diameter, two or three cryoprobes were placed within the center of the tumor, to ensure freezing of the entire mass. For masses with a long diameter of 6-10 $\mathrm{cm}$, the tumor was divided into two parts that were treated in turn, usually with an interval of 1 week. For masses with a long diameter of $>10 \mathrm{~cm}$, the tumor was divided into three parts treated at intervals of usually 1 week. The tracts formed were sealed with fibrin glue immediately after removal of the cryoprobes to ensure hemostasis.

In the DEB-TACE-Cryo group, percutaneous cryoablation was conducted under the guidance of USG and/or CT within the three weeks after DEB-TACE (median, 9 days; range, 3-18).

\section{Follow-up and response assessment}

To assess the success of the technique, contrastenhanced CT or magnetic resonance imaging (MRI) were performed 4-6 weeks after treatment. The efficacy evaluation was based on the modified response evaluation criteria in solid tumors (mRECIST). The imaging evaluation of the treatment result was made using a double blind method. Two radiologists, each with more than ten years of experience, independently interpreted the radiographic findings and consensus was reached on collective reading if their opinions were divided. If the outcome was considered a complete response, the treatment was considered successful and then follow up was conducted every three months. If residual active tumor remained, a second cryoablation cycle was performed after re-evaluation of the patient.

At each visit, HCC patients underwent radiographic and hematologic examinations. If distant multiple metastases were found, a symptomatic treatment or other palliative therapy was applied.

\section{Safety}

Enhanced CT and bedside ultrasonography were performed within $24 \mathrm{~h}$ of the ablation. The purpose of this first follow-up imaging was primarily to check for complications and not to evaluate treatment efficacy. Additionally, in order to evaluate the effect of the intervention on liver function, serum ALB, AST, ALT, and T.BIL levels were measured 7 and 30 days post-procedure. Complications within 30 days of treatment were evaluated in accordance with the criteria established by the Society of Interventional Radiology (SIR) and were graded according to the National Cancer Institute Common Terminology Criteria for Adverse Events Version 4.0 (CTCAE V4.0). Preoperative and postoperative pain scores were taken according to the Visual Analogue Score (VAS) criteria.

This study was censored on October 9, 2017.

\section{Statistical analysis}

All statistical analyses and all associated figures were performed and compiled using GraphPad software (GraphPad, San Diego, CA, USA). The continuous data, categorical data, and survival curves of the two groups were compared using the Mann Whitney, Chi-square, and Gehan-Breslow-Wilcoxon tests individually. Continuous data from the same group were compared using the paired $t$ test. The results were expressed as the mean \pm standard deviation. All statistical tests were two-sided and a $P$ value $<0.05$ was considered statistically significant.

\section{Abbreviations}

DEB, drug-eluting beads; TACE, transarterial chemoembolization; Cryo, cryoablation; HBsAg, hepatitis B surface ntigen; HBV, hepatitis B virus; HCV, hepatitis $\mathrm{C}$ virus; AJCC, American Joint Committee on Cancer; ECOG, Eastern Cooperative Oncology Group; AFP, $\alpha$-fetoprotein; ALT, alanine aminotransferase; AST, aspartate aminotransferase; T.BIL, total bilirubin; ALB, albumin.

\section{Author contributions}

Conception and design: Jian-Ying Zeng, Xiang-Hao Piao

Financial support: Jian-Guo Liu, Xiang-Hao Piao

Administrative support: Li-Zhi Niu, Ji-Bing Chen

Collection and assembly of data: Zhong-Yuan Zou, Qing-Feng Yang, Liang Zhou

Data analysis and interpretation: Jian-Ying Zeng, Zi-Lin Qin

Manuscript writing: All authors

J.-Y.Z. and X.-H.P. contributed equally to the manuscript.

\section{ACKNOWLEDGMENTS}

We would like to thank the native English speaking scientists of Elixigen Company (Huntington Beach, California) and Charron Cote for editing our manuscript. 


\section{CONFLICTS OF INTEREST}

The author(s) indicate no potential conflicts of interest.

\section{FUNDING}

This work was supported by the International Science Foundation of Affiliated Fuda Cancer Hospital, Jinan University [grant number Y2015-ZD-002] and the Tianhe District Science and Technology Project of China [grant number 201504KW009 and 201604KW025].

\section{REFERENCES}

1. Torre LA, Bray F, Siegel RL, Ferlay J, Lortet-Tieulent J, Jemal A. Global cancer statistics, 2012. CA Cancer J Clin. 2015; 65:87-108.

2. Bruix J, Sherman M. Management of hepatocellular carcinoma: an update. Hepatology. 2011; 53:1020-1022.

3. Bruix J, Sherman M. Management of hepatocellular carcinoma. Hepatology. 2005; 42:1208-1236.

4. Bruix J, Reig M, Sherman M. Evidence-based diagnosis, staging, and treatment of patients with hepatocellular carcinoma. Gastroenterology. 2016; 150:835-853.

5. Subramaniam S, Kelley RK, Venook AP. A review of hepatocellular carcinoma (HCC) staging systems. Chin Clin Oncol. 2013; 2:33.

6. Yamakado K, Nakatsuka A, Ohmori S, Shiraki K, Nakano T, Ikoma J, Adachi Y, Takeda K. Radiofrequency ablation combined with chemoembolization in hepatocellular carcinoma: Treatment response based on tumor size and morphology. J Vasc Interv Radiol. 2002; 13:1225-1232.

7. Katsanos K, Kitrou P, Spiliopoulos S, Maroulis I, Petsas T, Karnabatidis D. Comparative effectiveness of different transarterial embolization therapies alone or in combination with local ablative or adjuvant systemic treatments for unresectable hepatocellular carcinoma: a network metaanalysis of randomized controlled trials. PLoS One. 2017; 12:e0184597.

8. Wang ZJ, Wang MQ, Duan F, Song P, Liu FY, Chang ZF, Wang Y, Yan JY, Li K. Transcatheter arterial chemoembolization followed by immediate radiofrequency ablation for large solitary hepatocellular carcinomas. World J Gastroenterol. 2013; 19:4192-4199.

9. Peng ZW, Zhang YJ, Chen MS, Xu L, Liang HH, Lin XJ, Guo RP, Zhang YQ, Lau WY. Radiofrequency ablation with or without transcatheter arterial chemoembolization in the treatment of hepatocellular carcinoma: a prospective randomized trial. J Clin Oncol. 2013; 31:426-432.

10. Liu Z, Gao F, Yang G, Singh S, Lu M, Zhang T, Zhong Z, Zhang F, Tang R. Combination of radiofrequency ablation with transarterial chemoembolization for hepatocellular carcinoma: an up-to-date meta-analysis. Tumour Biol. 2014; 35:7407-7413.

11. Yoon SM, Lim YS, Won HJ, Kim JH, Kim KM, Lee HC, Chung YH, Lee YS, Lee SG, Park JH, Suh DJ. Radiotherapy plus transarterial chemoembolization for hepatocellular carcinoma invading the portal vein: longterm patient outcomes. Int J Radiat Oncol Biol Phys. 2012; 82:2004-2011.

12. Facciorusso A, Serviddio G, Muscatiello N. Local ablative treatments for hepatocellular carcinoma: an updated review. World J Gastrointest Pharmacol Ther. 2016; 7:477-489.

13. Lencioni R, de Baere T, Martin RC, Nutting CW, Narayanan G. Image-guided ablation of malignant liver tumors: Recommendations for clinical validation of novel thermal and non-thermal technologies - a western perspective. Liver Cancer. 2015; 4:208-214.

14. Song KD. Percutaneous cryoablation for hepatocellular carcinoma. Clin Mol Hepatol. 2016; 22:509-515.

15. Haen SP, Pereira PL, Salih HR, Rammensee HG, Gouttefangeas C. More than just tumor destruction: Immunomodulation by thermal ablation of cancer. Clin Dev Immunol. 2011; 2011:160250.

16. $\mathrm{Hu} \mathrm{KQ}$. Advances in clinical application of cryoablation therapy for hepatocellular carcinoma and metastatic liver tumor. J Clin Gastroenterol. 2014; 48:830-836.

17. Clavien PA, Kang KJ, Selzner N, Morse MA, Suhocki PV. Cryosurgery after chemoembolization for hepatocellular carcinoma in patients with cirrhosis. J Gastrointest Surg. 2002; 6:95-101.

18. Qian GJ, Chen H, Wu MC. Percutaneous cryoablation after chemoembolization of liver carcinoma: report of 34 cases. Hepatobiliary Pancreat Dis Int. 2003; 2:520-524.

19. Xu KC, Niu LZ, Zhou Q, Hu YZ, Guo DH, Liu ZP, Lan $\mathrm{B}, \mathrm{Mu}$ F, Li YF, Zuo JS. Sequential use of transarterial chemoembolization and percutaneous cryosurgery for hepatocellular carcinoma. World J Gastroenterol. 2009; 15:3664-3669.

20. Huang C, Zhuang W, Feng H, Guo H, Tang Y, Chen $\mathrm{H}$, Huang Y. Analysis of therapeutic effectiveness and prognostic factor on argon-helium cryoablation combined with transcatheter arterial chemoembolization for the treatment of advanced hepatocellular carcinoma. J Cancer Res Ther. 2016; 12:C148-C152.

21. Huang KB, Fan WZ, Zhang YY, Wang Y, Cui W, Li JP. [Transarterial chemoembolization combined with cryoablation for unresectable large hepatocellular carcinoma: a controlled study]. [Article in Chinese]. Zhonghua Yi Xue Za Zhi. 2016; 96:2978-2982.

22. van Malenstein H, Maleux G, Vandecaveye V, Heye S, Laleman W, van Pelt J, Vaninbroukx J, Nevens F, Verslype C. A randomized phase II study of drug-eluting beads versus transarterial chemoembolization for unresectable hepatocellular carcinoma. Onkologie. 2011; 34:368-376. 
23. Golfieri R, Giampalma E, Renzulli M, Cioni R, Bargellini I, Bartolozzi C, Breatta AD, Gandini G, Nani R, Gasparini D, Cucchetti A, Bolondi L, Trevisani F. Randomised controlled trial of doxorubicin-eluting beads vs conventional chemoembolisation for hepatocellular carcinoma. Br J Cancer. 2014; 111:255-264.

24. Facciorusso A, Di Maso M, Muscatiello N. Drug-eluting beads versus conventional chemoembolization for the treatment of unresectable hepatocellular carcinoma: a metaanalysis. Dig Liver Dis. 2016; 48:571-577.

25. Facciorusso A, Mariani L, Sposito C, Spreafico C, Bongini M, Morosi C, Cascella T, Marchiano A, Camerini T, Bhoori S, Brunero F, Barone M, Mazzaferro V. Drug-eluting beads versus conventional chemoembolization for the treatment of unresectable hepatocellular carcinoma. J Gastroenterol Hepatol. 2016; 31:645-653.

26. Malagari K, Pomoni M, Moschouris H, Kelekis A, Charokopakis A, Bouma E, Spyridopoulos T, Chatziioannou A, Sotirchos V, Karampelas T, Tamvakopoulos C, Filippiadis D, Karagiannis E, et al. Chemoembolization of hepatocellular carcinoma with HepaSphere 30-60 mum. Safety and efficacy study. Cardiovasc Intervent Radiol. 2014; 37:165-175.

27. Zurstrassen CE, Gireli LP, Tyng CJ, Bitencourt AG, Guimaraes MD, Barbosa PN, Santos Cavalcante AC, Matushita Junior JP, Amoedo MK, Coimbra FJ, Alves RC, Chojniak R. Safety and efficacy of HepaSphere 50-100 mum in the treatment of hepatocellular carcinoma. Minim Invasive Ther Allied Technol. 2017; 26:212-219.

28. Kucukay F, Badem S, Karan A, Ozdemir M, Okten RS, Ozbulbul NI, Kucukay MB, Unlu I, Bostanci EB, Akdogan $\mathrm{M}$. A single-center retrospective comparison of doxorubicinloaded HepaSphere transarterial chemoembolization with conventional transarterial chemoembolization for patients with unresectable hepatocellular carcinoma. J Vasc Interv Radiol. 2015; 26:1622-1629.

29. Sun JH, Zhou GH, Zhang YL, Nie CH, Zhou TY, Ai J, Zhu TY, Wang WL, Zheng SS. Chemoembolization of liver cancer with drug-loading microsphere 50-100mum. Oncotarget. 2017; 8:5392-5399. https://doi.org/10.18632/ oncotarget.14281.

30. Veltri A, Moretto P, Doriguzzi A, Pagano E, Carrara G, Gandini G. Radiofrequency thermal ablation (RFA) after transarterial chemoembolization (TACE) as a combined therapy for unresectable non-early hepatocellular carcinoma (HCC). Eur Radiol. 2006; 16:661-669.

31. Zhang L, Yin X, Gan YH, Zhang BH, Zhang JB, Chen Y, Xie XY, Ge NL, Wang YH, Ye SL, Ren ZG. Radiofrequency ablation following first-line transarterial chemoembolization for patients with unresectable hepatocellular carcinoma beyond the Milan criteria. BMC Gastroenterol. 2014; 14:11.

32. Zhao M, Wang JP, Pan CC, Li W, Huang ZL, Zhang L, Fang WJ, Jiang Y, Li XS, Wu PH. CT-guided radiofrequency ablation after with transarterial chemoembolization in treating unresectable hepatocellular carcinoma with long overall survival improvement. Eur J Radiol. 2012; $81: 2717-2725$

33. Orlacchio A, Bazzocchi G, Pastorelli D, Bolacchi F, Angelico M, Almerighi C, Masala S, Simonetti G. Percutaneous cryoablation of small hepatocellular carcinoma with US guidance and CT monitoring: initial experience. Cardiovasc Intervent Radiol. 2008; 31:587-594.

34. Varela M, Real MI, Burrel M, Forner A, Sala M, Brunet M, Ayuso C, Castells L, Montana X, Llovet JM, Bruix J. Chemoembolization of hepatocellular carcinoma with drug eluting beads: efficacy and doxorubicin pharmacokinetics. J Hepatol. 2007; 46:474-481.

35. Zou JH, Zhang L, Ren ZG, Ye SL. Efficacy and safety of cTACE versus DEB-TACE in patients with hepatocellular carcinoma: a meta-analysis. J Dig Dis. 2016; 17:510-517.

36. Bruix J, Sherman M, Llovet JM, Beaugrand M, Lencioni R, Burroughs AK, Christensen E, Pagliaro L, Colombo M, Rodes J. Clinical management of hepatocellular carcinoma. Conclusions of the Barcelona-2000 EASL conference. European Association for the Study of the Liver. J Hepatol. 2001; 35:421-430. 